\title{
Modeling of widely-linear quaternion valued systems using hypercomplex algorithms
}

\begin{abstract}
The data-driven optimal modeling and identification of widely-linear quaternion-valued synthetic systems is achieved by using a quaternion-valued gradient based algorithms. To account rigorously for the second-order statistics of the quaternion system, the quaternion least mean square (QLMS) and widely linear quaternion least mean square (WL-QLMS) were selected. The QLMS is shown to successfully model the quaternion-valued systems and the WL-QLMS is able to model both quaternion and widely-linear quaternion valued systems taking into account the full second-order statistics of the system. Analysis has proven that both algorithms are able to adapt to non-stationary nature of the systems. This approach is supported by simulations of various synthetic systems.
\end{abstract}

Keyword: Quaternion least mean square (QLMS); Quaternion second-order statistics; Nonstationary systems; System modeling; Widely linear model 